IRB-TH $1 / 97$

April 1997

\title{
Inclusive Charmed-Baryon Decays and Lifetimes
}

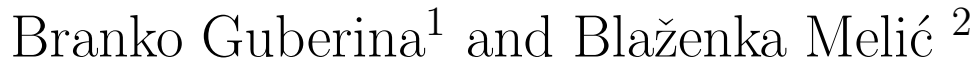 \\ Theoretical Physics Division, Rudjer Bošković Institute, P.O.B.1016 \\ HR-10001 Zagreb, Croatia
}

\begin{abstract}
We have quantitatively reanalyzed the inclusive charmed-baryon decays. New ingredients are the Voloshin preasymptotic effects in semileptonic decays and the Cabibbo-subleading contributions to both semileptonic and nonleptonic decays. It has been found that the Cabbibo-subleading Voloshin contribution essentially improves the theoretical semileptonic branching ratio of $\Lambda_{c}^{+}$, in agreement with experiment. The semileptonic branching ratios for $\Xi_{c}^{+}$and $\Omega_{c}^{0}$ are found to be large, i.e., of the order of $20 \%$. The lifetimes hierarchy is in a good qualitative and even quantitative agreement with experiment except for the $\Xi_{c}^{+}$lifetime, which is somewhat smaller than the experimental value. Future measurements, especially measurements of the semileptonic branching ratios for $\Omega_{c}^{0}, \Xi_{c}^{+}$ and $\Xi_{c}^{0}$ should be decisive for the check of this approach.
\end{abstract}

\footnotetext{
${ }^{1}$ E-mail: guberina@thphys.irb.hr

${ }^{2}$ E-mail: melic@thphys.irb.hr
} 


\section{Introduction}

Weak decays of charmed and bottom hadrons [1-4] are particularly simple in the limit of infinite heavy quark mass.

In reality, hadrons are bound states of heavy quark with light constituents (light quarks, gluons). The inclusion of soft degrees of freedom generates nonperturbative power corrections, an example of which is the destructive Pauli interference between the spectator light-quark and the light quark in $D^{+}$meson coming from the decay of the heavy quark [5-7].

Inclusive hadronic decay rates and lifetimes are calculated some time ago [6-9]. It has been found that the overall picture for charmed hadron lifetimes is qualitatively satisfactory. Lifetime hierarchy has been predicted for charmed baryons $[8,9]$, in qualitative agreement with present experiments [4]. It has also been shown $[6,7]$ that the Pauli interference essentially lengthens the lifetime of the $D^{+}$meson, thus being the main source of the $D^{0}-D^{+}$lifetime difference. The systematic OPE brings in extra operators of dimension 5, namely kinetic and chromomagnetic operators [10]. Their influence on charmed hadron lifetimes is rather moderate, because the main contribution comes from the four-quark operators. Using the current quark mass, $m_{c} \sim 1.4 \mathrm{GeV}$, the results for the charmed family are qualitatively good [1], provided one assumes that terms of higher dimension in the OPE drive the asymptotic (static) value of the decay constant, $F_{\text {meson }}$ close to the smaller value of the physical decay constant $f_{\text {meson }}$ only in meson decays, whereas in baryon decays that is not the case. In beauty decays, although everything was expected to work much better because of the large $b$-quark mass, a number of problems has remained unsolved $[2,3,11]$. In the first place, it is the discrepancy between the measured average semileptonic beauty meson branching ratio, which is somewhat smaller than the theoretical prediction. Second, the observed difference of the lifetimes of the $\Lambda_{b}$ baryon and of the $B$ meson is larger than expected. In view of these descrepancies, a radical phenomenological ansatz has been involved [11] with interesting consequences for charmed hadron decays. This approach, however, abandons the heavy-quark expansion and local duality, and requires the introduction [3] of a dominant destructive interference in the $\Xi_{c}^{+}$decay and destructive $W$-anihilation in $D_{s}$ decays - the requirements that could hardly be satisfied if the four-quark operators were a dominant source of preasymptotic effects.

Recently, Voloshin has shown [12] that preasymptotic effects are largely present in semileptonic decays of charmed baryons. A significant enhancement [3,12] of $\Gamma_{S L}\left(\Xi_{c}\right)$ and $\Gamma_{S L}\left(\Omega_{c}\right)$ is expected relative to $\Gamma_{S L}\left(\Lambda_{c}\right)$ and $\Gamma_{S L}\left(D^{0}\right)$ in order $1 / m_{c}^{3}$ owing to the constructive Pauli interference in $\Gamma_{S L}\left(\Xi_{c}, \Omega_{c}\right)$ among the $s$-quarks.

In this paper we present theoretical predictions on the $\Lambda_{c}^{+}, \Xi_{c}^{+}, \Xi_{c}^{0}$ and $\Omega_{c}^{0}$ semileptonic branching ratios and lifetimes. The lifetimes have already been treated in $[8,9]$. Here, we extend previous calculations, in which preasymptotic effects in nonleptonic decay rates were basically attributed to the Cabbibo leading operators of dimension 6. We make extension by including Voloshin's preasymptotic effects [12] in inclusive semileptonic decay rates. We calculate and add the Cabibbo subleading contribu- 
tions since it was claimed in the literature [13] that they might be important in some cases owing to the statistical factors. We show that the constructive Pauli interference, which appears in the semileptonic $\Lambda_{c}^{+}$decay at Cabbibo subleading level, is welcome because it enhances the theoretical value which is otherwise too small. Finally, we show that the inclusion of Voloshin's large preasymptotic effects in semileptonic decays does not destroy qualitative hierarchy of lifetimes, but improves it both qualitatively and quantitatively.

\section{Preasymptotic effects in inclusive decays}

The inclusive decay width of a hadron $H_{c}$, of the mass $M_{H_{c}}$, containing a $c$ quark can be written using the optical theorem as

$$
\Gamma\left(H_{c} \rightarrow f\right)=\frac{1}{2 M_{H_{c}}} 2 \operatorname{Im}\left\langle H_{c}|\hat{T}| H_{c}\right\rangle
$$

i.e. as the forward matrix element of the imaginary part of the transition operator $\hat{T}$

$$
\hat{T}=i \int d^{4} x T\left\{L_{e f f}(x), L_{e f f}^{\dagger}(0)\right\} .
$$

The effective weak Lagrangian $L_{e f f}(x)$ is given [1,3] as a sum of the semileptonic and nonleptonic part

$$
L_{e f f}=L_{e f f}^{S L}+L_{e f f}^{N L} .
$$

In the following we assume that the energy release in the decay of a $c$ quark is large enough so that momenta flowing through internal lines are also large and therefore justify the operator product expansion of the local operator (2). The result, widely discussed in the literature [1-3] is given by

$$
\begin{aligned}
\Gamma\left(H_{c} \rightarrow f\right) & =\frac{G_{\mathrm{F}}^{2} m_{c}^{5}}{192 \pi^{3}}|V|^{2} \frac{1}{2 M_{H_{c}}}\left\{c_{3}^{f}\left\langle H_{c}|\bar{c} c| H_{c}\right\rangle+c_{5}^{f} \frac{\left\langle H_{c}\left|\bar{c} g_{s} \sigma^{\mu \nu} G_{\mu \nu} c\right| H_{c}\right\rangle}{m_{c}^{2}}\right. \\
& \left.+\sum_{i} c_{6}^{f} \frac{\left\langle H_{c}\left|\left(\bar{c} \Gamma_{i} q\right)\left(\bar{q} \Gamma_{i} c\right)\right| H_{c}\right\rangle}{m_{c}^{3}}+O\left(1 / m_{c}^{4}\right)+\ldots\right\} .
\end{aligned}
$$

Here $c_{3}^{f}$ and $c_{5}^{f}$ are coefficient functions which depend on the particular final state. The coefficients $c_{3}^{f}$ are known at one-loop order and coefficients $c_{5}^{f}$ at tree level [10].

Let us calculate the semi-leptonic decay rates first. The main contribution is expected to come from the quark decay-type diagrams. When corrections $O\left(m_{c}^{-2}\right)$ are included, the contribution takes the form

$$
\Gamma_{S L}^{d e c}\left(H_{c}\right)=\frac{G_{F}^{2}}{192 \pi^{3}} m_{c}^{5}\left(1-\frac{1}{2} \frac{\mu_{\pi}^{2}\left(H_{c}\right)}{m_{c}^{2}}+\frac{1}{2} \frac{\mu_{G}^{2}\left(H_{c}\right)}{m_{c}^{2}}\right) F_{1}(x) .
$$


Here $\mu_{\pi}^{2}\left(H_{c}\right)$ and $\mu_{\mathrm{G}}^{2}\left(H_{c}\right)$ parametrize the matrix elements of the kinetic energy and the chromo-magnetic operators, respectively. They can be determined from the spectrum of charmed heavy hadrons $[14,15]$. It turns out that only $\mu_{G}^{2}\left(\Omega_{c}^{0}\right)$ is different from zero.

There is also the contribution of the dimension five operator

$$
\Gamma_{S L}^{G}\left(H_{c}\right)=\frac{G_{F}^{2}}{192 \pi^{3}} m_{c}^{5}\left(-2 \frac{\mu_{G}^{2}\left(H_{c}\right)}{m_{c}^{2}}\right) F_{2}(x) .
$$

We have included in the above expressions the phase space corrections $F_{1}(x)$ and $F_{2}(x)[16]$.

The semileptonic rate, as it has been shown by Voloshin [12], gets important and large contributions due to the preasymptotic effects. The result is given by

$$
\tilde{\Gamma}_{S L}=\frac{G_{F}^{2}}{12 \pi} m_{c}^{2}(4 \sqrt{\kappa}-1)|\psi(0)|^{2} .
$$

Here, $|\psi(0)|$ is the baryon wave function at the origin and $\kappa$ is a correction due to the hybrid renormalization of the effective Lagrangian. Hybrid renormalization is necessary since $|\psi(0)|^{2}$ is usually estimated in the effective quark models which are expected to make sense at the typical hadronic scales, $\mu=0.5 \sim 1 \mathrm{GeV}$. Therefore, it is necessary to evolve the effective Lagrangian from $m_{c}$ down to the scale $\mu$.

Total semileptonic rate is given by

$$
\Gamma_{S L}\left(H_{c}\right)=\Gamma_{S L}^{\text {dec }}\left(H_{c}\right)+\Gamma_{S L}^{G}\left(H_{c}\right)+\Gamma_{S L}^{\text {Voloshin }}\left(H_{c}\right),
$$

where

$$
\begin{aligned}
\Gamma_{S L}^{\text {Voloshin }}\left(\Lambda_{c}^{+}\right) & =s^{2} \tilde{\Gamma}_{S L}, \\
\Gamma_{S L}^{\text {Voloshin }}\left(\Xi_{c}^{+}\right) & =\xi c^{2} \tilde{\Gamma}_{S L}, \\
\Gamma_{S L}^{\text {oloshin }}\left(\Xi_{c}^{0}\right) & =\left(\xi c^{2}+s^{2}\right) \tilde{\Gamma}_{S L}, \\
\Gamma_{S L}^{V \text { oloshin }}\left(\Omega_{c}^{0}\right) & =\frac{10}{3} \xi c^{2} \tilde{\Gamma}_{S L} .
\end{aligned}
$$

Here $s^{2}$ and $c^{2}$ are abbreviations for $\sin ^{2} \theta_{c}$ and $\cos ^{2} \theta_{c}$, and $\theta_{c}$ is the Cabibbo angle.

We have kept the Cabibbo-suppressed contributions because the preasymptotic effects are expected to be very large, and, therefore they might be a significant correction to $\Gamma_{S L}^{\text {dec }}\left(H_{c}\right)$. Also, we have introduced the parameter $\xi$ which is the ratio of the matrix elements of the operators $\left(\bar{c}_{L} \gamma_{\mu} s_{L}\right)\left(\bar{s}_{L} \gamma^{\mu} c_{L}\right)$ and $\left(\bar{c}_{L} \gamma_{\mu} q_{L}\right)\left(\bar{q}_{L} \gamma^{\mu} c_{L}\right)$, where $q$ is $d$ or $u$ quark. $S U(3)$ symmetry-breaking effects, measured by $\xi$ are not expected to exceed $30 \%$. It is very difficult to reliably estimate the value of $\xi$, although certain hints can be made using different hadronic models. For example, the hadronic models used in [9] would suggest $\xi>1$, but in view of the fact that we lack reliable models such a conclusion might be premature. We shall not rely on such estimates in later discussions, but prefer to treat $\xi$ as a fitting parameter.

So far, only semileptonic branching ratio of $\Lambda_{c}^{+}$has been measured with reasonable accuracy

$$
B R\left(\Lambda_{c}^{+} \rightarrow e X\right)=(4.5 \pm 1.7) \% .
$$


By inspection of Eq.(9) one notes that except for $\Lambda_{c}^{+}$, all baryons receive potentially large Voloshin's contributions at the Cabibbo-leading level. Therefore, one expects, as pointed by Voloshin [12], significantly larger semileptonic ratio for $\Xi_{c}^{+}, \Xi_{c}^{0}$ and $\Omega_{c}^{0}$. Besides, the contribution to $\Lambda_{c}^{+}$semileptonic branching ratio, although Cabbibosuppressed by $\sin ^{2} \theta_{c}$, might be an important correction to the decay diagram. This is welcome, since the decay diagram is not large enough to explain the experimental branching ratio [16].

The calculation of the nonleptonic decay rate closely follows the semileptonic ones. The lepton pair is substituted by a quark pair, and Wilson coefficients $c_{ \pm}$ change their values because of the renormalization. The contributions coming from the $c$-quark decay-type diagrams (including $O\left(m_{c}^{-2}\right)$ corrections) and from the dimension five operator are of the form

$$
\begin{aligned}
\Gamma_{N L}^{d e c}\left(H_{c}\right) & =\frac{G_{F}^{2}}{192 \pi^{3}} m_{c}^{5}\left(c_{-}^{2}+2 c_{+}^{2}\right)\left(1-\frac{1}{2} \frac{\mu_{\pi}^{2}\left(H_{c}\right)}{m_{c}^{2}}+\frac{1}{2} \frac{\mu_{G}^{2}\left(H_{c}\right)}{m_{c}^{2}}\right) F_{1}(x), \\
\Gamma_{N L}^{G}\left(H_{c}\right) & =-\frac{G_{F}^{2}}{192 \pi^{3}} m_{c}^{3}\left(8 c_{+}^{2}-2 c_{-}^{2}\right) \mu_{G}^{2}\left(H_{c}\right) .
\end{aligned}
$$

The dominant contribution is expected to come from the preasymptotic effects. They are given as

$$
\begin{aligned}
\Gamma^{e x} & =\frac{G_{F}^{2}}{2 \pi} m_{c}^{2}\left[c_{-}^{2}+\frac{2}{3}(1-\sqrt{\kappa})\left(c_{+}^{2}-c_{-}^{2}\right)\right]|\psi(0)|^{2}, \\
\Gamma_{-}^{i n t} & =\frac{G_{F}^{2}}{2 \pi} m_{c}^{2}\left[-\frac{1}{2} c_{+}\left(2 c_{-}-c_{+}\right)-\frac{1}{6}(1-\sqrt{\kappa})\left(5 c_{+}^{2}+c_{-}^{2}-6 c_{+} c_{-}\right)\right]|\psi(0)|^{2}, \\
\Gamma_{+}^{i n t} & =\frac{G_{F}^{2}}{2 \pi} m_{c}^{2}\left[\frac{1}{2} c_{+}\left(2 c_{-}+c_{+}\right)-\frac{1}{6}(1-\sqrt{\kappa})\left(5 c_{+}^{2}+c_{-}^{2}+6 c_{+} c_{-}\right)\right]|\psi(0)|^{2} .
\end{aligned}
$$

The result of the calculation of the nonleptonic rates is

$$
\begin{aligned}
\Gamma_{N L}\left(\Lambda_{c}^{+}\right) & =\Gamma_{N L}^{d e c}\left(\Lambda_{c}^{+}\right)+c^{2} \Gamma^{e x}+\Gamma_{-}^{i n t}+s^{2} \Gamma_{+}^{i n t}, \\
\Gamma_{N L}\left(\Xi_{c}^{+}\right) & =\Gamma_{N L}^{d e c}\left(\Xi_{c}^{+}\right)+\xi s^{2} \Gamma^{e x}+\Gamma_{-}^{i n t}+\xi c^{2} \Gamma_{+}^{i n t}, \\
\Gamma_{N L}\left(\Xi_{c}^{0}\right) & =\Gamma_{N L}^{d e c}\left(\Xi_{c}^{-}\right)+\left(c^{2}+\xi s^{2}\right) \Gamma^{e x}+\left(\xi c^{2}+s^{2}\right) \Gamma_{+}^{i n t}, \\
\Gamma_{N L}\left(\Omega_{c}^{0}\right) & =\Gamma_{N L}^{d e c}\left(\Omega_{c}^{0}\right)+\Gamma_{N L}^{G}\left(\Omega_{c}^{0}\right)+\xi s^{2} \frac{10}{3} \Gamma^{e x}+\xi c^{2} \frac{10}{3} \Gamma_{+}^{i n t} .
\end{aligned}
$$

Here we have not taken into account mass corrections, because they are completely negligible.

By inspection of the results one sees that Cabibbo suppressed contribution only slightly changes the overall results. The right pattern depends on the value of $m_{c}$ and $|\psi(0)|^{2}$. However, for $\kappa=1, \Gamma_{+}^{i n t}$ is always larger than $\Gamma_{-}^{i n t}$. This conclusion holds even if hybrid logarithms are taken into account. Therefore, the $\Gamma_{+}^{\text {int }}$ will dominate the nonleptonic $\Xi_{c}^{+}$decay rate, as far as $\xi \simeq 1$. Since experimentally $\Xi_{c}^{+}$ has the largest lifetime this rate should be relatively small.

For the determination of the baryon wave function we use estimates of the references $[9,1]$, i.e the relation for the ratio of the squares of the meson and baryon wave 
functions which is derived using the constituent quark model developed by De Rujula et al. [17]. There appear 'effective' quark masses, $m_{c}^{*} \simeq 1.5 \mathrm{GeV}, m_{u}^{*} \simeq 0.35 \mathrm{GeV}$ and quarks are bound by a nonrelativistic potential which is modified by hyperfine interactions.

Following the approach of Ref.[1], in the expression for the baryonic wave function we shall use the static value $F_{D}$ instead of the physical decay constant $f_{D}$ for the reasons given below. This leads to

$$
\left|\psi^{\Lambda_{c}^{+}}(0)\right|^{2}=\frac{3\left(M_{\Sigma_{c}^{+}}-M_{\Lambda_{c}^{+}}\right)}{\mu_{G}^{2}(D)} m_{u}^{*}\left(\frac{1}{12} M_{D} F_{D}^{2} \kappa^{-4 / 9}\right)
$$

The importance of the value of $\left|\psi^{\Lambda_{c}^{+}}(0)\right|^{2}$ is obvious since the differences in decay widths/lifetimes are presumably generated mostly by the operators of dimension 6 (four-quark operators) and are therefore proportional to $F_{D}^{2} / m_{c}^{2}$, which indeed vanishes as $1 / m_{c}^{3}$, since $F_{D}$ behaves as $m_{c}^{-1 / 2}$ for $m_{c} \rightarrow \infty$.

It has been argued [1], on a more intuitive basis, that in order to be consistent, one should use the static value $F_{D}$ in the calculations of the baryon decay. For meson decays, however, one should assume that the role of higher dimension terms is not negligible, and consequently, the physical (measured) constant $f_{D}$ should be used in calculations.

These arguments [1] are based on the fact that in meson decays one uses the factorization which necessarily brings into game the physical decay constant $f_{D}$, whereas in baryon decays this is not the case. Since there is no proof of this ansatz, one should take it as an attempt to disentangle the overall normalization of the mesonic matrix elements from the baryonic ones. In fact, it is known from previous calculations that, for example, the destructive Pauli interference in the $D^{+}$ meson decay has reasonable values when the bag model wave functions are used [6], while the charmed baryon hierarchy was qualitatively well described by using nonrelativistic quark models [9]. In other words, in order to achieve agreement with experimental data, different normalization of matrix elements had to be used for meson and baryon decays.

\section{Semileptonic branching ratios and lifetime hierarchy - results and discussions}

Our choice of 'central' values of parameters roughly follows the set of values of Blok and Shifman [1]. For $\Lambda_{Q C D}=300 \mathrm{MeV}$, the Wilson coefficients are $c_{+}=0.734$, $c_{-}=1.856$.

Our central value for the charmed quark mass is $m_{c}=1.4 \mathrm{GeV}$. However, in Table 1. we show the results for $m_{c}=1.35 \mathrm{GeV}$ for comparison. There is a 


\begin{tabular}{|l|c|c|c|}
\hline \hline & \multicolumn{2}{|c|}{ RESULTS } & \multirow{2}{*}{$\begin{array}{c}\text { EXP. DATA } \\
\end{array}$} \\
\cline { 2 - 3 } & $\left.m_{c}=1, \Lambda_{Q C D}=300 \mathrm{MeV}, \mu=1 \mathrm{GeV}\right)$ & $m_{c}=1.35 \mathrm{GeV}$ & \\
\hline \hline & \multicolumn{2}{|c|}{ Lifetimes in units $10^{-13} \mathrm{~s}$} & \\
\hline \hline$\tau\left(\Lambda_{c}^{+}\right)$ & 2.03 & 2.18 & $2.06 \pm 0.12$ \\
\hline$\tau\left(\Xi_{c}^{+}\right)$ & 2.42 & 2.70 & $3.50 \pm 0.70$ \\
\hline$\tau\left(\Xi_{c}^{0}\right)$ & 0.87 & 0.94 & $0.98 \pm 0.23$ \\
\hline$\tau\left(\Omega_{c}^{0}\right)$ & 0.58 & 0.63 & $0.64 \pm 0.20$ \\
\hline \hline & \multicolumn{2}{|c|}{ Lifetime ratios } & \\
\hline \hline$\tau\left(\Xi_{c}^{+}\right) / \tau\left(\Lambda_{c}^{+}\right)$ & 1.19 & 1.23 & $1.69 \pm 0.35$ \\
\hline$\tau\left(\Xi_{c}^{0}\right) / \tau\left(\Lambda_{c}^{+}\right)$ & 0.43 & 0.43 & $0.47 \pm 0.11$ \\
\hline$\tau\left(\Omega_{c}^{0}\right) / \tau\left(\Lambda_{c}^{+}\right)$ & 0.28 & 0.29 & $0.31 \pm 0.09$ \\
\hline$\tau\left(\Xi_{c}^{+}\right) / \tau\left(\Xi_{c}^{0}\right)$ & 2.76 & 2.87 & $3.57 \pm 1.10$ \\
\hline & Semileptonic decay rates in units $\mathrm{ps}{ }^{-1}$ & \\
\hline \hline$\Gamma_{S L}\left(\Lambda_{c}^{+}\right)$ & 0.209 & 0.177 & $0.225 \pm 0.085$ \\
\hline$\Gamma_{S L}\left(\Xi_{c}^{+}\right)$ & 1.010 & 0.918 & \\
\hline$\Gamma_{S L}\left(\Xi_{c}^{0}\right)$ & 1.053 & 0.958 & - \\
\hline$\Gamma_{S L}\left(\Omega_{c}^{0}\right)$ & 2.954 & 2.718 & - \\
\hline \hline & Semileptonic branching ratios in $\%$ & \\
\hline \hline$B R_{S L}\left(\Lambda_{c}^{+}\right)$ & 4.2 & 3.8 & $4.5 \pm 1.7$ \\
\hline$B R_{S L}\left(\Xi_{c}^{+}\right)$ & 24.5 & 24.8 & - \\
\hline$B R_{S L}\left(\Xi_{c}^{0}\right)$ & 9.2 & 9.0 & - \\
\hline$B R_{S L}\left(\Omega_{c}^{0}\right)$ & 17.4 & 17.3 & - \\
\hline \hline
\end{tabular}

Table 1: Predictions for semileptonic branching ratios and lifetimes of charmed baryons given for two values of the charmed quark mass $m_{c}$.

controversy [15] over the value of $\mu_{\pi}^{2}$, which varies in the range [2]

$$
\mu_{\pi}^{2}(B) \simeq-\lambda_{1}=(0.3 \pm 0.2) \mathrm{GeV}^{2} .
$$

In our calculations we use the lower value, $\mu_{\pi}^{2}=0.1 \mathrm{GeV}^{2}$. However, we also check that the larger value $\mu_{\pi}^{2}=0.5 \mathrm{GeV}^{2}$ only slightly changes the result in semileptonic branching ratios, but has almost no effect on lifetimes. The chromomagnetic operator contributes only to $\Omega_{c}^{0}$ decays [1]; we use the value

$$
\mu_{G}^{2}\left(\Omega_{c}^{0}\right)=0.182 G e V^{2} .
$$

Following [1], we use the following input to obtain the central value in Eq.(14): $F_{D}=400 \mathrm{MeV}, m_{u}^{*}=350 \mathrm{MeV}, M_{\Sigma_{c}^{*}}-M_{\Lambda_{c}^{+}}=400 \mathrm{MeV}$ (static value) and $M_{D}=$ $1870 \mathrm{MeV}$. For $\mu_{G}^{2}(D)$, we use the value $\mu_{G}^{2}(D)=0.4 \mathrm{GeV}^{2}$. Then, for $\kappa=1$, Eq.(14) gives our central value for the baryon wave function

$$
\left|\psi^{\Lambda_{c}^{+}}(0)\right|_{\mu=m_{c}}^{2}=0.0262 \mathrm{GeV}^{3} .
$$




\begin{tabular}{|c|c|c|c|}
\hline & \multicolumn{2}{|c|}{$\begin{array}{c}\text { RESULTS } \\
\left(m_{c}=1.4 \mathrm{GeV}, \Lambda_{Q C D}=300 \mathrm{MeV}, \mu=1 \mathrm{GeV}\right)\end{array}$} & \multirow{2}{*}{$\begin{array}{l}\text { EXP. DATA } \\
{[4]}\end{array}$} \\
\hline & $\xi=1$ & $\xi=0.75$ & \\
\hline & \multicolumn{2}{|c|}{ Lifetimes in units $10^{-13} \mathrm{~s}$} & \\
\hline$\overline{\tau\left(\Lambda_{c}^{+}\right)}$ & 2.03 & 2.03 & $\overline{20.06 \pm 0.12}$ \\
\hline$\tau\left(\Xi_{c}^{+}\right)$ & 2.42 & 3.41 & $3.50 \pm 0.70$ \\
\hline$\tau\left(\Xi_{c}^{0}\right)$ & 0.87 & 0.98 & $0.98 \pm 0.23$ \\
\hline$\tau\left(\Omega_{c}^{0}\right)$ & 0.58 & 0.76 & $0.64 \pm 0.20$ \\
\hline e e & \multicolumn{2}{|c|}{ Semileptonic branching ratios in \% } & \\
\hline 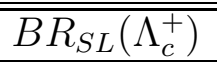 & $\overline{4.2}$ & $\overline{4.2}$ & $4.5 \pm 1.7$ \\
\hline$B R_{S L}\left(\Xi_{c}^{+}\right)$ & 24.5 & 27.2 & $\overline{-}$ \\
\hline$B R_{S L}\left(\Xi_{c}^{0}\right)$ & 9.2 & 8.2 & $\overline{-}$ \\
\hline$B R_{S L}\left(\Omega_{c}^{0}\right)$ & 17.4 & 17.3 & $\overline{-}$ \\
\hline
\end{tabular}

Table 2: Predictions for charmed baryons in dependence of the parameter $\xi$.

Our numerical results are presented in Tables 1-2. The left set of numbers in the Tables are numerical results obtained for our central values discussed before, and for $m_{c}=1.4 \mathrm{GeV}, m_{s}=150 \mathrm{MeV}, \mu=1 \mathrm{GeV}$ and $\xi=1$. The agreement with available experimental data is very good, except for the lifetime of $\Xi_{c}^{+}$, where theoretically predicted value is smaller than in experiment (see Table 1). The same problem with $\Xi_{c}^{+}$persists if one calculates the ratio of lifetimes (in such a way significantly reducing the uncertainty coming from the wave-function value). However, the semileptonic branching ratio for $\Lambda_{c}^{+}$is in excellent agreement with the experimental value, showing clearly that preasymptotic effects of Voloshin's type, although at the Cabibbo suppressed level, significantly improve the theoretical value.

For $\Lambda_{Q C D}=200 \mathrm{MeV}$ the results are almost not affected except the $\Lambda_{c}^{+}$lifetime which grows by $20 \%$, becoming so unpleasantly large. However, one should keep in mind that such modest discrepances are expected since in charmed baryon decays we are far away from the asymptotic limit.

In Table 1 we also display results of the calculations for the smaller value of the current quark mass, $m_{c}=1.35 \mathrm{GeV}$. Again, the results are not very sensitive to this variation, although the agreement with experiment is slightly improved, especially for $\Xi_{c}^{+}$decays.

In Table 2 we have presented the results of calculations for the specific choice of the parameter $\xi, \xi=0.75$, compared with the results obtained for $\xi=1$. As discussed above, we allow $\xi$ to have a value different from 1, treating it as a free parameter. Fitting $\xi$ roughly to the value needed to bring the $\Xi_{c}^{+}$lifetime into agreement with experiment gives $\xi \simeq 0.75$. Fitting $\xi$ basically means to fit the constructive interference term $\Gamma_{+}^{\text {int }}$ for Cabibbo favoured decays. The simple fit of one lifetime would of course, not make much progress. However, $\Gamma_{+}^{\text {int }}$ enters also the decay rates of other baryons, $\Xi_{c}^{0}$ and $\Omega_{c}^{0}$, at the same Cabibbo level. Besides, 

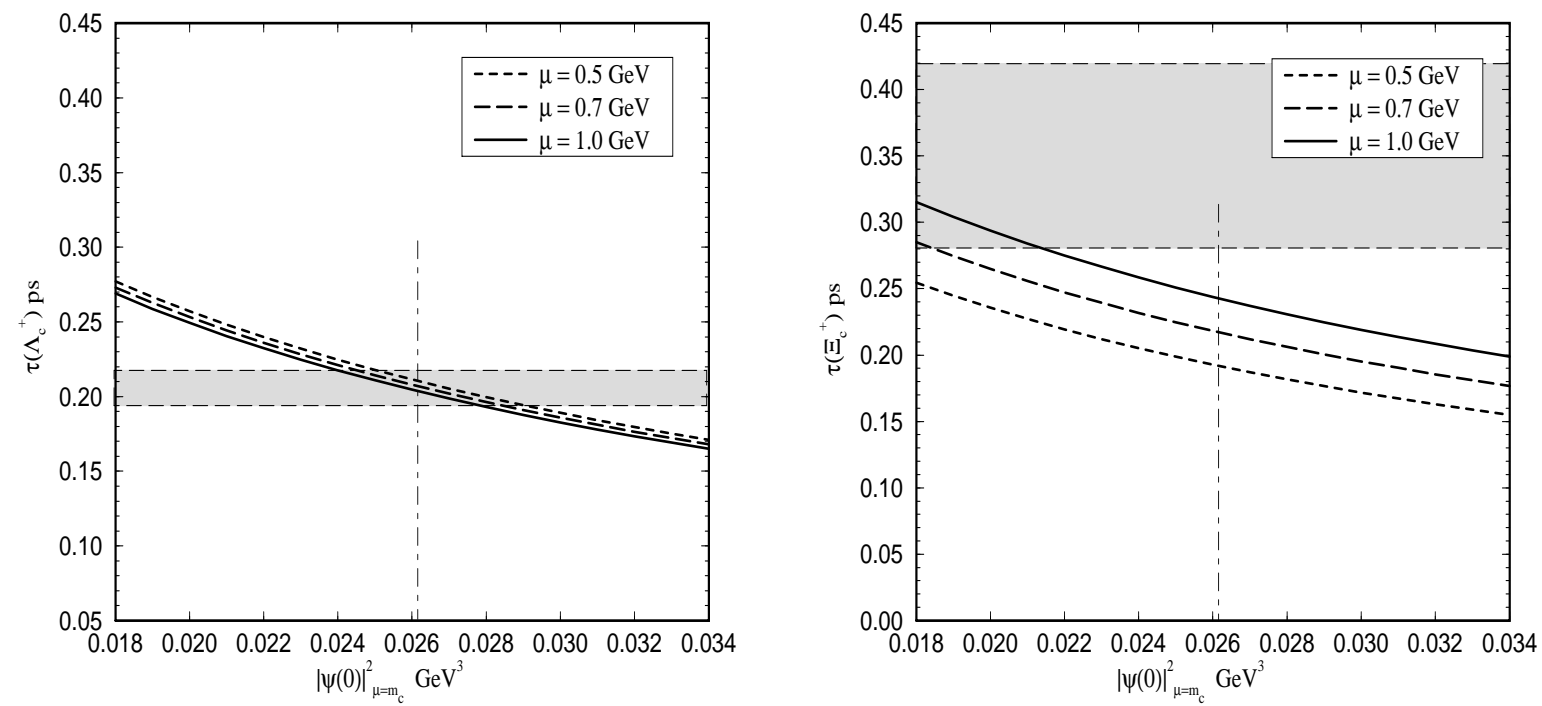

Figure 1: Lifetimes of $\Lambda_{c}^{+}$and $\Xi_{c}^{+}$as a function of the square of the baryon wave function $|\psi(0)|^{2}$, given for three values of the hybrid renormalization point $\mu$. The shaded areas are the experimentally allowed regions. The dot-dashed vertical line is a value of $|\psi(0)|^{2}$ used in Tables 1 to 2 . The results are obtained using $m_{c}=1.4 \mathrm{GeV}$, $\Lambda_{Q C D}=300 \mathrm{MeV}, \mu_{\pi}^{2}=0.1 \mathrm{GeV}^{2}$.

the same factor $\xi$ enters Voloshin's contributions to semileptonic decay rates, again at the same Cabibbo level. Therefore, any trivial fit to $\Xi_{c}^{+}$could at the same time worsen the results for other particles. However, interestingly enough, here this is not the case. Although the fit $\xi=0.75$ brings the $\Xi_{c}^{+}$lifetime into perfect agreement between theory and experiment, it does not spoil the agreement between theory and experiment for both semileptonic BR's and lifetimes of other particles.

Next we study the dependence of lifetimes on the square of the baryon wave function $|\psi(0)|^{2}$ and show the results in Fig.1 and 2. We vary the value $|\psi(0)|^{2}$ inside a factor of 2 in the range

$$
0.018 \mathrm{GeV}^{3} \leq\left|\psi^{\Lambda_{c}^{+}}(0)\right|_{\mu=m_{c}}^{2} \leq 0.034 \mathrm{GeV}^{3},
$$

with a central value given by (17).

It is interesting to note that the lifetimes of $\Lambda_{c}^{+}$and $\Xi_{c}^{+}$are very sensitive to the value of $\left|\psi^{\Lambda_{c}^{+}}(0)\right|^{2}$, Fig.1. On the other hand, the other decays are not so sensitive, and are consistent with experiment even for the lower value of $\left|\psi_{c}^{\Lambda_{c}^{+}}(0)\right|^{2}$ given in (18), Fig.2. Therefore, one could easily bring the lifetimes of $\Xi_{c}^{+}, \Xi_{c}^{0}$ and $\Omega_{c}^{0}$ to agreement with experiment simply by using a smaller value of $\left|\psi^{\Lambda_{c}^{+}}(0)\right|^{2}$. This would enlarge the $\Lambda_{c}^{+}$lifetime and introduce a descrepancy between theory and experiments. However, our analysis of lifetimes, as discussed above, shows that the $\Xi_{c}^{+}$lifetime exhibits a peculiar behavior - strong $\mu$-dependence and strong $|\psi(0)|^{2}$ dependence. Furthermore, the central value of $\left|\psi^{\Lambda_{c}^{+}}(0)\right|^{2}$, given in (36), gives a good 

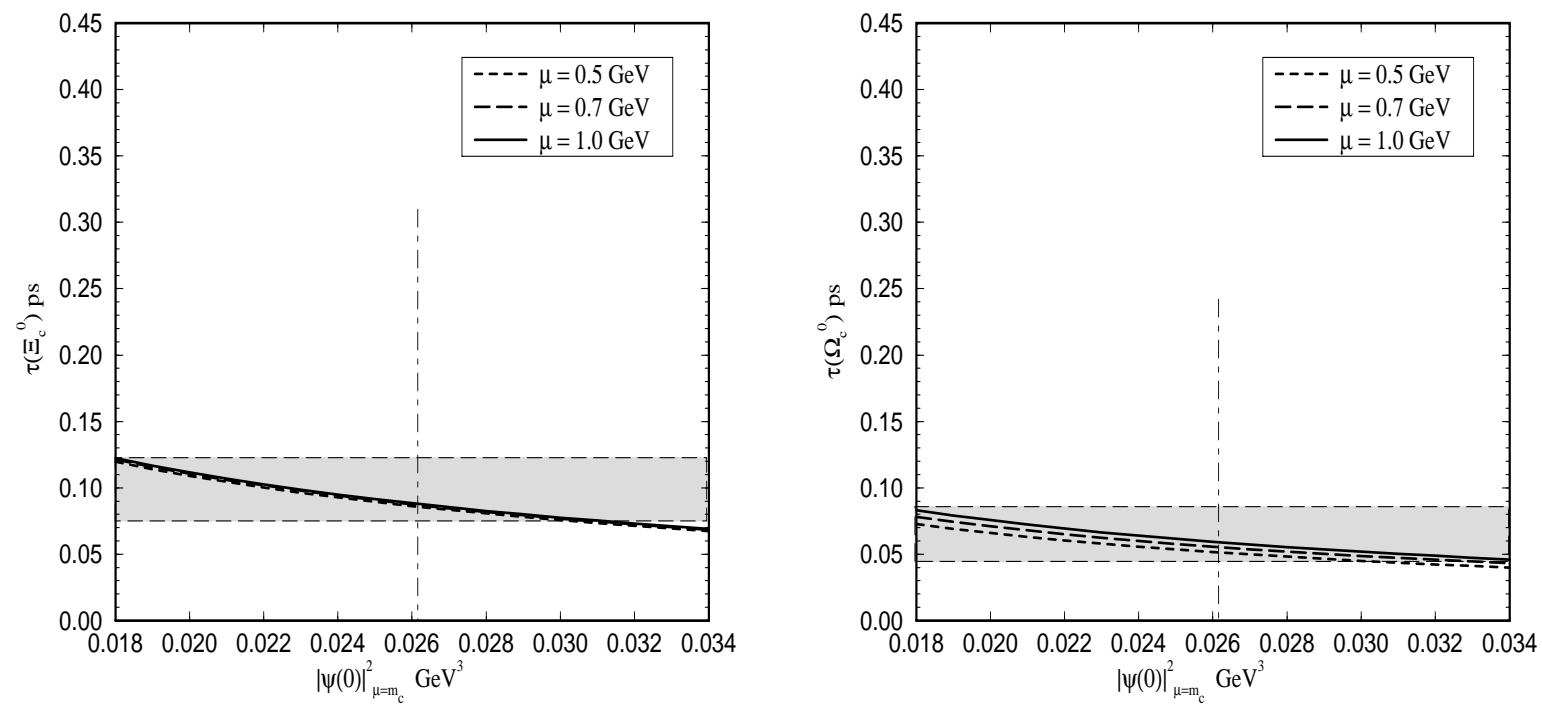

Figure 2: Lifetimes of $\Xi_{c}^{0}$ and $\Omega_{c}^{0}$ as a function of the square of the baryon wave function $|\psi(0)|^{2}$ given for three values of the hybrid renormalization point $\mu$. The shaded areas are the experimentally allowed regions. The dash-dotted vertical line is a value of $|\psi(0)|^{2}$ used in Tables 1 to 2 . The results are obtained using $m_{c}=1.4 \mathrm{GeV}$, $\Lambda_{Q C D}=300 \mathrm{MeV}, \mu_{\pi}^{2}=0.1 \mathrm{GeV}^{2}, \mu_{G}^{2}\left(\Omega_{c}^{0}\right)=0.182 \mathrm{GeV}^{2}$.

semileptonic branching ratio of $\Lambda_{c}^{+}$, which we discuss next.

As discussed in the preceding section, baryons receive Voloshin's large interference contributions at the Cabibbo leading level. Their role is obvious from Table 1 , where a certain hierarchy of semileptonic BR's is strongly pronounced:

$$
B R_{S L}\left(\Lambda_{c}^{+}\right)<B R_{S L}\left(\Xi_{c}^{0}\right)<B R_{S L}\left(\Omega_{c}^{0}\right)<B R_{S L}\left(\Xi_{c}^{+}\right)
$$

It is in the numerical range from 4.5 to 25 percent. We consider prediction (19) as a crucial test of the approach presented here, for the following reasons: Voloshin's interference effects, being proportional to $|\psi(0)|^{2}$ are necessarily large, because one needs a large $|\psi(0)|^{2}$ in order to reproduce experimental values of lifetimes. If one finds experimentally that all semileptonic BR's are of the order of $B R_{S L}\left(\Lambda_{c}^{+}\right)$, this will mean that the interference effect in semileptonic decays is negligible, and that, therefore, the preasymptotic effects in (12) are unlikely to be responsible for the experimentally evident hierarchy of lifetimes.

In addition to this very clear prediction, Voloshin's interference effect helps to improve the theoretical value of the $\Lambda_{c}^{+}$semileptonic branching ratio. It appears at the Cabibbo suppressed level and acts as a correction to the main contribution coming from the decay diagram. It has been known for a long time that the quark decay mechanism cannot explain the semileptonic branching ratio of $\Lambda_{c}^{+}$, if one uses the current quark mass $m_{c} \sim 1.4 \mathrm{GeV}$ in (5). An effective mass of the order $1.6-1.7 \mathrm{GeV}$ is actually needed $[1-3,11]$. 

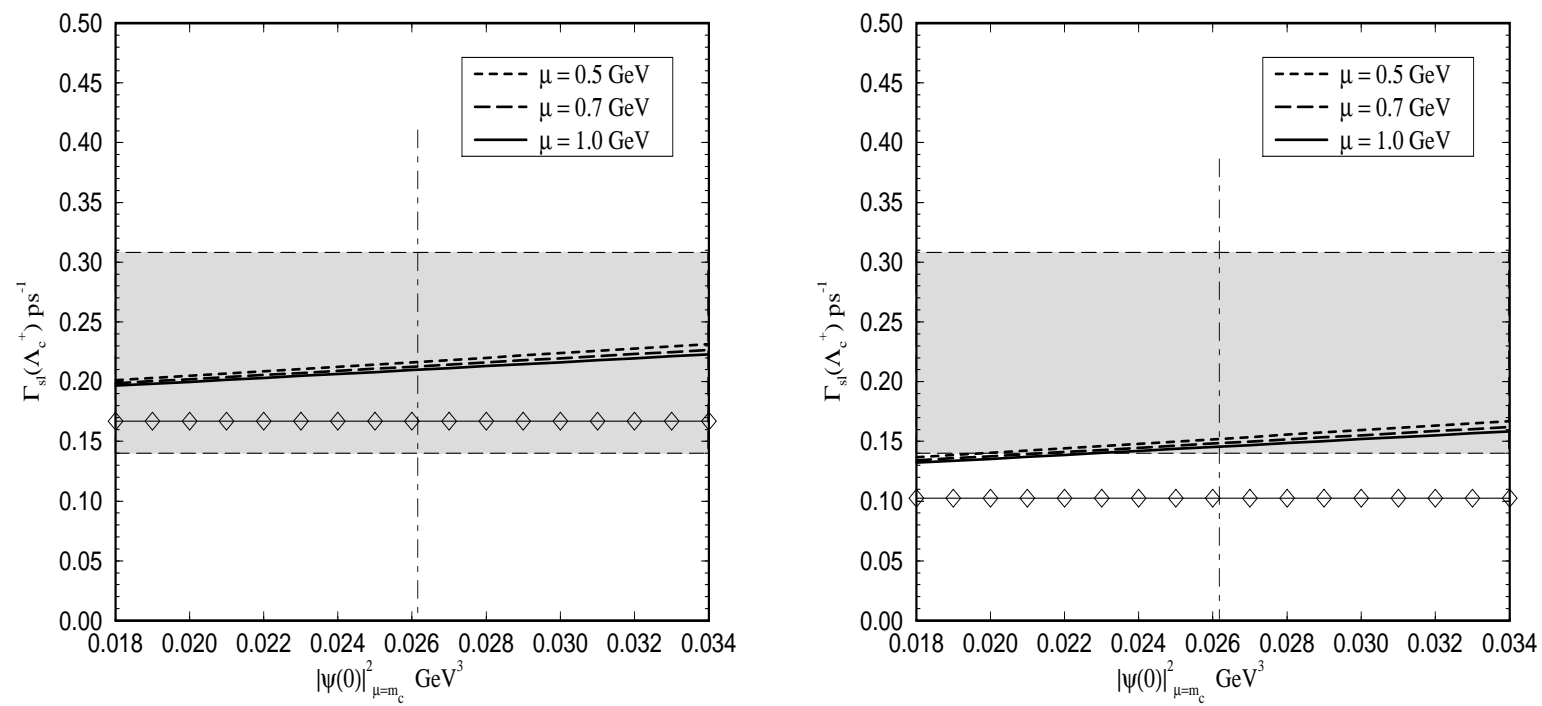

Figure 3: Semileptonic $\Lambda_{c}^{+}$-decay rate for three values of the hybrid renormalization point $\mu$ is given as a function of the square of the baryon wave function $|\psi(0)|^{2}$. On the left picture finite $\alpha_{s}$-contributions are not included. The vertical dot-dashed line denotes the 'central' value in (17). The shaded area is the experimentally allowed region. $\diamond$ denotes the semileptonic decay rate without Voloshin's contributions. The other parameter values used are $m_{c}=1.4 \mathrm{GeV}, \Lambda_{Q C D}=300 \mathrm{MeV}, \mu_{\pi}^{2}=0.1 \mathrm{GeV}^{2}$.

In Fig. 3 we show the semileptonic branching ratio for $\Lambda_{c}^{+}$as a function of $|\psi(0)|^{2}$, for two cases, namely with and without finite $\alpha_{s}$-corrections. It is quite clear that the interference effect is welcome, because it brings the theoretical value close to experiment. In all calculations, presented in Tables 1-2, the finite $\alpha_{s}$-corrections have not been taken into account because they are not known for all kinds of preasymptotic contribution. However, the right figure in Fig.3 shows the semileptonic branching ratio with finite $\alpha_{s}$-corrections included. Obviously, without the interference effect the theoretical value is outside the experimentally allowed region.

\section{Conclusions}

In this paper we have performed an analysis of inclusive semileptonic branching ratios and lifetimes for the charmed baryon family. New ingredients in this analysis are the inclusion of preasymptotic effects in semileptonic decays and the inclusion of Cabibbo suppressed contributions. In the calculations we have used the input parameters determined by QCD, thus following the approach of Blok and Shifman [1], i.e. we have avoided the introduction of "effective" parameters, such as effective 
charmed quark mass, effective hadron mass instead of quark mass, etc. In this way we have tried to test quark-hadron duality using our present knowledge of OPE and QCD up to the level of introducing the baryon wave function, $\left|\psi^{\Lambda_{c}^{+}}(0)\right|$ as a measure of the strength of the dominant preasymptotic effects. Having fixed the charmed quark mass to be approximately $m_{c} \sim 1.4 \mathrm{GeV}$, the semileptonic branching ratios and lifetimes of charmed baryons depend essentially on the square of the baryon wave function, $\left|\psi^{\Lambda_{c}^{+}}(0)\right|^{2}$, which, in spirit of the above considerations may be regarded as a fitting parameter. It is a pleasent discovery that a rough fit of $\left|\psi^{\Lambda_{c}^{+}}(0)\right|^{2}$ agrees very well with the Blok-Shifman estimate [1].

Our analysis leads to the following conclusions:

i) The inclusion of Voloshin's large preasymptotic effects leads to following predictions: The semileptonic branching ratios of $\Xi_{c}^{+}, \Xi_{c}^{0}$, and $\Omega_{c}^{0}$ are significantly larger than the semileptonic branching ratios of $\Lambda_{c}^{+}$with a hierarchy already given in Eq.(19):

$$
B R_{S L}\left(\Lambda_{c}^{+}\right)<B R_{S L}\left(\Xi_{c}^{0}\right)<B R_{S L}\left(\Omega_{c}^{0}\right)<B R_{S L}\left(\Xi_{c}^{+}\right) .
$$

The inclusion of the Cabibbo suppresed interference effect in the semileptonic decay rate of $\Lambda_{c}^{+}$enhances it and brings the branching ratio to agreement with experiment. ii) The change in semileptonic decay rates which is due to interference effects significantly helps to obtain very good qualitative and even quantitive results for the lifetimes with the same hierarchy

$$
\tau\left(\Omega_{c}^{0}\right)<\tau\left(\Xi_{c}^{0}\right)<\tau\left(\Lambda_{c}^{+}\right)<\tau\left(\Xi_{c}^{+}\right)
$$

as predicted in $[8,9]$. The predicted lifetime of $\Xi_{c}^{+}$appears to be somewhat smaller than the experimental value. We do not consider that as a problem, since we are far away from the asymptotic limit and it would be premature to expect that higher order terms in OPE are really negligible.

iii) Concerning the results as obtained and shown in Tables 1-2, one may conclude that quark-hadron duality works suprisingly well for the charmed baryon family.

After completion of this work we have learned of a recent paper of Cheng [18] where Voloshin's type of corrections were considered in a different context, in a more phenomenological way, introducing the effective charmed-quark mass, substitution of the universal quark mass by the particular physical hadron mass, etc. We believe that our results, compared with the results of Cheng [18], are more consistent and more reliable.

\section{Acknowledgement}

This work was supported by the Ministry of Science and Technology of the Republic of Croatia under the contract Nr. 00980102. 


\section{References}

1. B. Blok and M. Shifman: "Lifetimes of Charmed Hadrons Revised - Facts and Fancy." in Proc. of the Workshop on the Tau-Charm Factory, p.247, Marbella, Spain, 1993, eds. J. Kirkby and R. Kirkby (Editions Frontiers, Gif- sur-Yvette, 1994), TPI-MINN-93/55-T, UMN-TH-1227/93.

2. M. Neubert: "B Decays and the Heavy-Quark Expansion", preprint CERNTH/97-24 and hep-ph/9702375, to appear in 'Heavy Flavors II', eds. A.J. Buras and M. Lindner.

3. I.I. Bigi: "Heavy Quark Expansions for Inclusive Heavy-Flavor Decays and the Lifetimes of Charm and Beauty Hadrons", invited lecture given at HQ96 'Heavy Quarks at Fixed Target', St. Goar, Germany, Oct. 3.-6., 1996, UNDHEP- 96-BIG06 and hep-ph/9612293.

4. Review of Particle Properties: Phys. Rev. D54 (1996).

5. B. Guberina, S. Nussinov, R.D. Peccei and R. Rückl: Phys. Lett. B89 (1979) 111.

6. N. Bilić, B. Guberina and J. Trampetić: Nucl. Phys. B248 (1984) 261.

7. M. Shifman and M. Voloshin: Yad. Fiz. 41 (1985) 463 [ Sov. J. Nucl. Phys. 41 (1985) 120].

8. M. Shifman and M. Voloshin: ZhETF 91 (1986) 1180 [ JETP 64 (1986) 698].

9. B. Guberina, R. Rückl and J. Trampetić: Z. Phys. C33 (1986) 297.

10. M. Neubert: Phys. Rep. 245 (1994) 259; Int. J. Mod. Phys. A11 (1996)4173.

11. G. Altarelli, G. Martinelli, S. Petrarca and F. Rapuano: Phys. Lett. B382 (1996) 409. 
12. M. Voloshin: Phys. Lett. B385 (1996) 369.

13. V. Gupta and K.V.L. Sarma: Int. J. Mod. Phys. A5 (1990) 879.

14. I. Bigi, M. Shifman and N. Uraltsev: "Aspects of Heavy Quark Theory", TPIMINN-97/02-T, UMN-TH-1528-97, UND-HEP-97-BIG01 and hep-ph/9703290.

15. M. Neubert: "Theory of Inclusive $B$ Decays", invited talk at the $4^{\text {th }}$ KEK Topical Conference on Flavor Physics, KEK, Japan, Oct. 29-31, 1996, CERNTH/9-19 and hep-ph/9702310.

16. I. Bigi: "The QCD Perspective on Lifetimes of Heavy-Flavour Hadrons", preliminary version of the Physics Report paper, UND-HEP-95-BIG02 and hep$\mathrm{ph} / 9508408$.

17. A. de Rujula, H. Georgi and S. Glashow: Phys. Rev. D12 (1975) 147; J.L. Cortes and J. Sanchez-Guillen: Phys. Rev. D24 (1981) 2982.

18. H.Y. Cheng: "A Phenomenological Analysis of Heavy Hadron Lifetimes", IPASTP-02-97 and hep-ph/9704260. 